\title{
Pulmonary Nocardiosis: Unusual Presentation in Intensive Care Unit
}

\author{
Ashok Kumar Singh, Aditya Shukla, R. Bajwa, Roshni Agrawal', Neha Srivastwa \\ Departments of Critical Care Medicine and 'Microbiology, Regency Hospital, Kanpur, Uttar Pradesh, India
}

\section{Abstract}

Pulmonary nocardiosis is a rare disease, which mainly affects immunocompromised hosts such as uncontrolled diabetics; HIV patients; and patients on immunosuppressive therapies such as anticancer drugs, systemic steroids, and transplant recipients. The diagnosis is often missed and delayed, resulting in inappropriate treatment and thus higher mortality. We are reporting a case of pulmonary nocardiosis with acute presentation in an immune-competent host, who presented with community-acquired pneumonia to the Intensive Care Unit. Clinical expertise with multiple high-end and interventional investigations timely confirmed the case as pulmonary nocardiosis. Conservative management with medications led to her complete recovery.

Keywords: Immune-competent host, linezolid, pulmonary nocardiosis

\section{INTRODUCTION}

Pulmonary nocardiosis is a chronic disease occurring in immunocompromised patients mimicking tuberculosis, mycotic infection, or malignancy. It is caused by aerobic actinomycetes which usually originate from soil. Clinical and radiological manifestations are just like tuberculosis or malignancy, which include pneumonia, infiltrative, or nodular lesions which may be accompanied by cavitation, abscess formation, pleural effusions, or empyema and sometimes mass-like lesions. Acute presentation of nocardiosis is very rare, especially in immunocompetent host. Co-trimoxazole is the first-line antimicrobial therapy for nocardiosis, but carbapenems and linezolid may be second-line drugs if the patient is intolerance to sulfa drugs. If the disease is not diagnosed in early stage and it becomes disseminated to multiple organs such as brain, lymphoreticular system, and musculoskeletal system, then outcome is very poor with high mortality of about $70 \%-80 \%$.

\section{Case Report}

A 36-year-old female patient presented to the emergency department with a complaint of fever, hemoptysis, left-sided chest pain, and breathlessness for 7 days. She is not having any significant history of diabetes, malignancy, and drug intake. On

\begin{tabular}{|l|l|}
\hline \multicolumn{3}{|c|}{ Access this article online } \\
\hline Quick Response Code: & Website: \\
& www.ijccm.org \\
\cline { 2 - 2 } & \\
\end{tabular}

clinical examination, her vitals were as follows: temperature $102^{\circ} \mathrm{F}$, respiratory rate $32 / \mathrm{min}$, blood pressure $100 / 76 \mathrm{~mm}$ of $\mathrm{Hg}$, heart rate $108 / \mathrm{min}$, and $\mathrm{SpO}_{2}$ of $92 \%$ in room air. On examination of the respiratory system, breath sound was decreased on the left side of the chest, significant crepitation was present on left infraclavicular, axillary and interscapular area, rest systems were within normal limits.

As the patient was having active hemoptysis and chest pain, she was transferred to Medical Intensive Care Unit for the purpose of monitoring and observations. After resuscitation of the patient, she was evaluated for coronary artery diseases by electrocardiography, two-dimensional echo, and cardiac markers, which were found to be normal. Tests for HIV, hepatitis $\mathrm{B}$ virus surface antigen, $\mathrm{HCV}$, and autoimmune profile were negative. Chest X-ray revealed a mass lesion in the left upper and mid-zone of the lung, resembling neoplasia [Figure 1a].

Sputum examination did not reveal any pathogenic organism in Gram staining and culture reports. In this case, fiberoptic

Address for correspondence: Dr. Ashok Kumar Singh, Department of Critical Care Medicine, Regency Hospital, A-2, Sarvodaya Nagar, Kanpur - 208 005, Uttar Pradesh, India. E-mail: dr_ashok_rhl@yahoo.com

This is an open access article distributed under the terms of the Creative Commons Attribution-NonCommercial-ShareAlike 3.0 License, which allows others to remix, tweak, and build upon the work non-commercially, as long as the author is credited and the new creations are licensed under the identical terms.

For reprints contact: reprints@medknow.com

How to cite this article: Singh AK, Shukla A, Bajwa R, Agrawal R, Srivastwa N. Pulmonary nocardiosis: Unusual presentation in Intensive Care Unit. Indian J Crit Care Med 2018;22:125-7. 
bronchoscopy is the best option for tissue diagnosis, but it was avoided due to active hemoptysis and respiratory distress. Her computed tomography (CT) scan thorax with contrast study revealed a large mass lesion with contract enhancement [Figure 1b], leading to high suspicion of malignancy. We planned a CT-guided fine-needle aspiration cytology (FNAC) from the lesion. The sample which was received by CT-guided FNAC was sent for cytological examination as well as for Gram staining and Ziehl-Neelsen staining and aerobic culture. It was found to be negative for malignant cells. Gram staining showed the presence of Gram-positive branching filaments with coccoid elements suggestive of Nocardia species [Figure 2a]. Conventional Ziehl-Neelsen staining for acid-fast bacilli was negative; however, modified Ziehl-Neelsen staining using 1\% sulfuric acid showed partially acid-fast branching filaments [Figure $2 \mathrm{~b}$ ]. Blood agar plate after incubation of $48 \mathrm{~h}$ at $37^{\circ} \mathrm{C}$ revealed dry whitish-to-tan colonies, which on further incubation showed the typical raised, chalky white appearance with the characteristic earthy odor of Nocardia species [Figure 2c]. Staining of smears from culture also showed filamentous bacteria.

She was empirically put on the third-generation cephalosporin and azithromycin. After a confirm diagnosis of pulmonary nocardiosis, cotrimoxazole was started, but she had rashes all over the body. Antibiotic treatment was changed to imipenem for 10-day duration followed by oral linezolid. Chest X-ray revealed gradual resolution of pneumonia in the

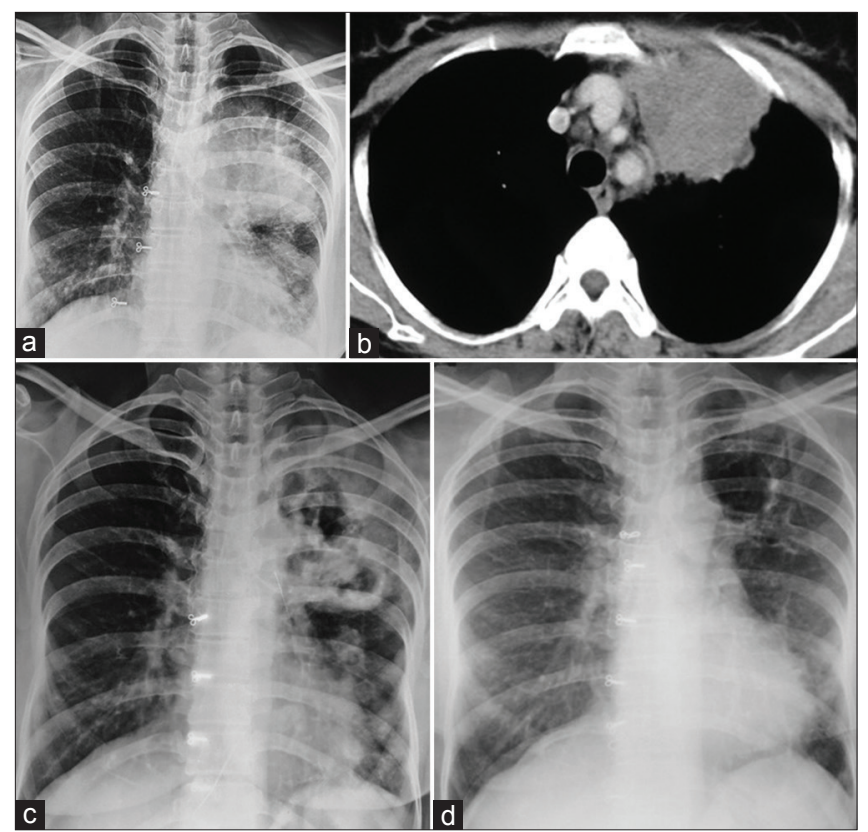

Figure 1: (a) Chest X-ray revealed a mass lesion in the left upper and mid-zone, resembling neoplastic in nature. (b) Computed tomography scan thorax with contrast study, which revealed large mass-like lesion with contract enhancement. (c) Chest $X$-ray revealed a resolving mass lesion in the left upper and mid-zone, resembling cavitating pneumonia. (d) Patient responded well to this therapy and had complete resolution of the left upper lobe lesion in follow-up chest X-ray form of cavitation [Figure 1c]. The patient responded well to this therapy and had complete resolution of the left upper lobe lesion in follow-up chest X-ray done after 6 months [Figure 1d].

\section{Discussion}

Nocardiosis, caused by Gram-positive, aerobic actinomycetes, is a rare and serious infection mainly affecting immunocompromised patients, with approximately $65 \%$ of cases occurring in individuals with some compromise of host defense systems. ${ }^{[1]}$ Human disease is most commonly caused by Nocardia asteroids. ${ }^{[2]}$ The manifestations of nocardiosis can be solely pulmonary $(75 \%-80 \%)$, cutaneous, or neurological (44\%), but virtually any organ system may be involved. ${ }^{[3]}$ The risk of pulmonary and disseminated disease is greater among persons with deficient cell-mediated immunity especially that associated with lymphoma, transplantation, systemic steroid, or AIDS.

Clinical features are relatively nonspecific with a chronic course in $70 \%$ before diagnosis. ${ }^{[4]}$ Pulmonary nocardiosis tends to present typically as a subacute pneumonia, the symptoms appearing over the course of several days or weeks. Acute presentation such as dyspnea, pleuritic pain, and hemoptysis are less common. ${ }^{[5]}$ There is variable radiological presentation as consolidation, well-circumscribed nodules, large, multiloculated abscesses, or cavities ${ }^{[6]}$ The major differentials in diagnosis are pneumonia, tuberculosis, bronchogenic carcinoma, or lung abscess. Pulmonary nodules with multiple cavitations can easily mimic actinomycosis, septic emboli, metastasis, sarcoidosis, Wegener's granulomatosis, or other fungal infections. ${ }^{[7]}$

The diagnosis should always be based on isolation of organism in respiratory secretions which can be sputum or bronchial

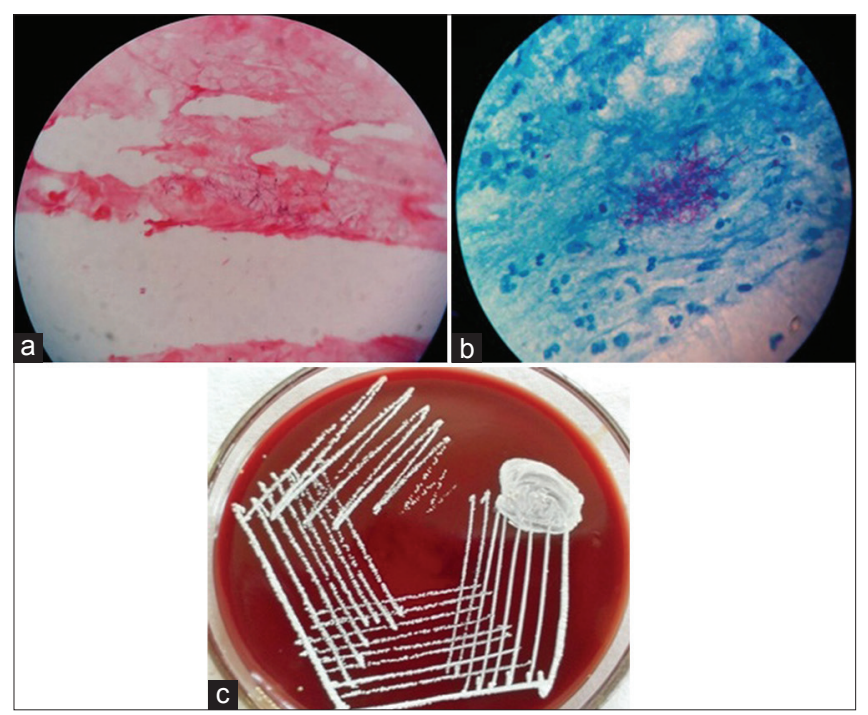

Figure 2: (a) Gram stain of a Nocardia species, demonstrating delicate, beaded, branching filaments. (b) Nocardia seen under high power after modified Ziehl-Neelsen staining. (c) Nocardia colonies on blood agar 
washings. Management includes antimicrobial therapy in all cases with surgical drainage wherever needed. Cotrimoxazole is the drug of choice which can be given alone or in combination with other drugs such as imipenem, amikacin, third-generation cephalosporins, or minocycline in serious cases. Disseminated nocardiosis has a poor prognosis with a mortality rate of $>85 \%$ in immunocompromised hosts, but in healthy patients this is about $15 \%-20 \%{ }^{\left[{ }^{[8]}\right.}$

\section{Conclusion}

Pulmonary nocardiosis is a chronic disease affecting mainly a host who is having defective immune system. Acute presentation is very rare in clinical practice. We have presented this case because this was acute presentation in an immunocompetant host which was diagnosed timely and managed accordingly with a positive outcome. If a patient is not having significant toxemia and negative for malignancy, we must think about these uncommon organisms like Nocardia.

\section{Declaration of patient consent}

The authors certify that they have obtained all appropriate patient consent forms. In the form the patient(s) has/have given his/her/their consent for his/her/their images and other clinical information to be reported in the journal. The patients understand that their names and initials will not be published and due efforts will be made to conceal their identity, but anonymity cannot be guaranteed.

\section{Financial support and sponsorship}

Nil.

\section{Conflicts of interest}

There are no conflicts of interest.

\section{RefEREnCES}

1. Frazier AR, Rosenow EC $3^{\text {rd }}$, Roberts GD. Nocardiosis. A review of 25 cases occurring during 24 months. Mayo Clin Proc 1975;50:657-63.

2. Wadhwa V, Rai S, Kharbanda P, Kabra S, Gur R, Sharma VK, et al. A fatal pulmonary infection by Nocardia brasiliensis. Indian J Med Microbiol 2006;24:63-4.

3. Sud S, Buxi T, Anand I, Rohatgi A. Case series: Nocardiosis of the brain and lungs. Indian J Radiol Imaging 2008;18:218-21.

4. Menéndez R, Cordero PJ, Santos M, Gobernado M, Marco V. Pulmonary infection with Nocardia species: A report of 10 cases and review. Eur Respir J 1997;10:1542-6.

5. Betriu C. Infections of Nocardia. Enferm Infecc Microbiol Clin 1997;15:154-60.

6. Feigin DS. Nocardiosis of the lung: Chest radiographic findings in 21 cases. Radiology 1986;159:9-14.

7. Hwang JH, Koh WJ, Suh GY, Chung MP, Kim H, Kwon OJ, et al. Pulmonary nocardiosis with multiple cavitary nodules in a HIV-negative immunocompromised patient. Intern Med 2004;43:852-4.

8. Lederman ER, Crum NF. A case series and focused review of nocardiosis: Clinical and microbiologic aspects. Medicine (Baltimore) 2004;83:300-13. 